\title{
Les microentreprises féminines et la pandémie de COVID-19 à Brazzaville en République du Congo : simples stratégies ou innovations sociales?
}

\author{
Mathias Marie Adrien Ndinga ${ }^{a}$
}

\begin{abstract}
RÉSUMÉ. L'objectif du présent travail est d'identifier les stratégies développées par les femmes entrepreneures engagées dans les microentreprises et de vérifier si celles-ci constituent des innovations sociales ou de simples stratégies. Pour ce faire, des récits de vie ont été recueillis, pendant la période du confinement pandémique, notamment au mois d'avril 2020. L'analyse de ces récits a montré que les stratégies mises en place par ces femmes sont ambidextres, c'est-à-dire qu'elles associent aussi bien les stratégies d'exploitation que d'exploration. Les stratégies d'exploitation portent sur les déplacements, sur la flexibilité dans la gestion de la main-d'œuvre et sur l'ajustement des jours et des horaires de travail afin de maintenir un certain niveau d'activité en pleine crise pandémique. Les secondes stratégies d'exploration, quant à elles, portent sur la recherche d'une nouvelle clientèle et sur le développement de nouveaux produits pendant cette période. Les critères de Bund et ses collaborateurs (2013) permettent de montrer que les objectifs visés par les stratégies d'exploration constituent bien des innovations sociales. Enfin, ces résultats ont des implications sur les stratégies d'adaptation et de survie des microentreprises des femmes en période de crise.
\end{abstract}

\begin{abstract}
The objective of this paper is to identify the strategies developed by businesswomen engaged in micro-entreprises and to verify whether these constitute social innovations or mere strategies. To do this, life stories were collected during the period of pandemic confinement, particularly in April 2020. The analysis of these narratives shows that the strategies implemented by these women are ambidextrous, i.e. combine both exploitation and exploration strategies. The exploitation strategies relate to travel, to flexibility in workforce management and to adjusting working days and hours to maintain a certain level of activity in the midst of the COVID-19 crisis. On the other hand, the exploration strategies focus on finding a new clientele and on developing new products during this period. The criteria of Bund and his colleagues (2013) show that the objectives targeted by the exploration strategies are indeed social innovations. Finally, these results have repercussions on the adaptation and survival strategies of women's microenterprises in times of crisis.
\end{abstract}

\section{Introduction}

Dans les pays en développement, les microentreprises, en général, et les microentreprises féminines en particulier, jouent un rôle important dans l'élargissement et le maintien du tissu économique et social. En effet, les microentreprises féminines sont devenues, au fil des années, des acteurs importants dans la sphère d'influence de l'économie domestique (OCDE, 2004; Lesteven, 1985). Bien que considérées à la fois comme les entreprises les plus dynamiques et les plus vulnérables, elles sont essentielles à l'amélioration du bien-être des populations, car elles jouent un rôle important dans le développement humain, dans la réduction de la pauvreté et dans la croissance économique durable.

Au Congo, les microentreprises féminines représentent 20,44\% des microentreprises du pays (MPMEASI et INS., 2017) dont le rôle dans le développement est capital. Elles permettent de réduire la pauvreté, les inégalités et le chômage tout comme elles aident les populations à répondre à

\footnotetext{
a Maitre de conférences, agrégé, Faculté des sciences économiques et LARES, Université Marien Ngouabi, Brazzaville, République du Congo
} 
leurs besoins fondamentaux et soutiennent les ménages à revenu modeste aussi bien en milieu urbain qu'en milieu rural. De plus, les microentreprises profitent à l'économie nationale en raison du potentiel d'utilisation des ressources locales dont les matières premières locales, de la mobilisation de l'épargne locale, de l'offre de possibilités de travail indépendant et de la possibilité de former des travailleurs semi-qualifiés par l'apprentissage (Asare, Akuffobea, Quaye et Atta Antwi, 2015).

Ces microentreprises développent des stratégies de survie qui les rendent souvent ambidextres en période de crise. Le concept d'ambidextérité (ou ambidextrie) renvoie à des microentreprises qui poursuivent des objectifs duals souhaitables, mais concurrents qui les obligent à structurer et à gérer leurs activités, de manière innovante (Gulati et Puranam, 2009; Smith et Lewis, 2011). Aussi, la généralisation de cette tendance dans les entreprises a donné lieu à une importante littérature autour du concept d'ambidextrie, laquelle porte essentiellement sur la compréhension de la manière dont les entreprises parviennent à un équilibre simultané entre les objectifs concurrentiels, par exemple l'efficacité et la flexibilité, l'alignement et l'adaptabilité, l'exploitation et l'exploration (Prieto et Pérez Santana, 2012; Turner, Swart et Maylor, 2013; Ahammad, Mook Lee, Malul et Shohama, 2015; Zimmermann, Raisch et Birkinshaw, 2015; Hahn, Pinkse, Preuss et Figge, 2016).

Dès lors, la présente réflexion répond à un double questionnement. Primo, les stratégies de survie des microentreprises dirigées par des femmes en pleine crise de pandémie les rendent-elles ambidextres? Secundo, certaines de ces stratégies de survie peuvent-elles être considérées comme des innovations sociales? Le rôle que jouent les femmes entrepreneures dans la réduction de la pauvreté en s'aidant de ces activités génératrices de revenus les amènerait à développer l'ambidextrie pour survivre à la crise pandémique. La nécessité de développer de nouveaux produits et d'explorer de nouveaux marchés les pousserait à faire des innovations sociales.

Cet article comprend quatre sections. La première section est consacrée à la revue de la littérature, tandis que la deuxième porte sur la méthodologie de travail et la présentation des récits de vie. La troisième section est axée sur la discussion autour des récits de vie. Enfin, une discussion est présentée, suivie de la conclusion et des implications politiques.

\section{Revue de littérature}

La littérature sur les stratégies de survie des entreprises est abondante. Ces stratégies peuvent être classées en deux catégories: celles axées sur le positionnement et celles portant sur les ressources.

\section{Stratégies axées sur le positionnement}

Les stratégies axées sur le positionnement, mises en évidence dans l'article fondateur de Porter (1980), considèrent que les microentreprises doivent s'adapter à leur environnement en évaluant les forces concurrentielles ${ }^{1}$ qui agissent dans cet environnement afin de déterminer la façon dont elles peuvent être compétitives.

Les stratégies de repli (Michael et Robbins, 1998; Dedee et Vorhies, 1998) impliquent la réduction des coûts d'exploitation et le désinvestissement des actifs non essentiels. Ces stratégies semblent être les approches les plus courantes adoptées par les entreprises pour faire face aux conditions de récession, en particulier, à court terme. Les analystes font état de désinvestissements d'entreprise, de fermetures d'établissement, de réductions d'emploi ainsi que de réductions des dépenses dans un large éventail d'activités, y compris la R-D, le marketing et la formation des employés.

\section{Stratégies axées sur les ressources}

Les stratégies axées sur les ressources, mises en évidence dans l'article précurseur de Penrose (1989) et développées par Rumelt (1984), Wernerfelt (1984) et Barney (1991), mettent l'accent sur le fait que l'avantage concurrentiel d'une microentreprise réside principalement dans les ressources dont elle dispose et dans la manière dont elle peut les utiliser pour obtenir un avantage concurrentiel. C'est dans cette optique que s'inscrivent les stratégies d'investissement qui impliquent des dépenses pour l'innovation et la diversification des marchés (Bryan et Farrell, 2008; Lynn, 2009).

\section{Stratégies ambidextres}

D'autres travaux (Teece, 2007; Eisenhardt et Martin, 2000) ont apporté un nouveau concept dans les stratégies axées sur les ressources. Il s'agit 
de la capacité dynamique, qui désigne la capacité d'une microentreprise à s'adapter à un environnement changeant ainsi qu'à étendre ses ressources et ses compétences. Les stratégies ambidextres (He et Wong, 2004; Raisch et Birkinshaw, 2008) qui combinent retranchement et investissement, s'inscrivent dans cette lignée. Ce sont ces stratégies qui seront placées au cœur de notre analyse.

En effet, la littérature consacrée à l'ambidextérité se concentre, souvent, sur la recherche d'un équilibre et d'une simultanéité entre l'exploitation et l'exploration. À cet égard, les travaux empiriques s'orientent dans trois directions. La première est constituée des travaux qui mettent l'accent sur l'ambidextérité structurelle, partielle ou réciproque (Chebbi, Yahiaoui, Vrontis et Thrassoua, 2015). La deuxième direction est empruntée par les travaux qui placent au centre de la stratégie ambidextérité la capacité des dirigeants à combiner les activités d'exploitation et d'exploration (Zimmermann et collab., 2015). La troisième direction est le fait des travaux qui placent au centre de l'ambidextérité le comportement des employés dans la conduite des activités d'exploitation et d'exploration (Hahn et collab., 2016).

\section{Ambidextérité structurelle, partielle et réciproque}

En ce qui concerne l'ambidextérité structurelle, partielle et réciproque, il convient de relever que cette stratégie consiste à développer des mécanismes structurels qui rendent possible soit l'alternance, soit la coïncidence entre les périodes d'exploitation et d'exploration. Plusieurs travaux (Gupta, Smith et Shalley, 2006; Cao, Gedajlovic et Zhange, 2009; Turner et collab., 2013) ont montré que cette stratégie améliore la performance des entreprises. Seulement, les mécanismes par lesquels cette stratégie est implantée font encore l'objet de débats. March (1991), dans son article séminal, a mis l'accent sur la nécessité de gérer les compromis pour trouver un équilibre approprié entre les deux activités (Farjoun, 2010; Turner et collab., 2013).

\section{Ambidextérité axée sur la capacité à combiner}

$\mathrm{Au}$ sujet de l'ambidextérité qui s'appuie sur la capacité des dirigeants à combiner les activités d'exploitation et d'exploration, Zimmermann et ses collègues (2015), relèvent que l'entreprise est performante lorsque le comportement des cadres est orienté vers la combinaison de ces deux types de ces deux types d'activités. Dans cette optique, la stratégie ambidextre est conduite de façon descendante (du haut vers le bas). Les cadres supérieurs développent un point de vue sur ce qui doit être fait et mettent en place les dispositions organisationnelles appropriées pour le réaliser. Ainsi, ils développent une intention stratégique globale d'ambidextérité, qui est, ensuite mise en œuvre par les autres employés (O'Reilly et Tushman, 2008; Momva van den Bosch et Volberda, 2009).

\section{Ambidextérité axée sur le comportement des employés}

Enfin, il y a la stratégie d'ambidextérité qui met l'accent sur le comportement des employés dans la conduite des activités d'exploitation et d'exploration (Hahn et collab., 2016). Selon Wooldridge et Floyd (1990), les employés peuvent jouer un rôle actif dans le développement et la promotion de processus de renouvellement stratégique. Plusieurs travaux (Prieto et Pérez antana, 2012; Ahammad et collab., 2015) montrent que les flux de connaissances ascendants permettent aux cadres supérieurs de mieux comprendre les changements concernant les technologies, les produits et les marchés, ce qui peut les inciter à revoir leurs décisions stratégiques.

\section{Commentaires}

Cette revue de la littérature suscite quelques commentaires. Le premier tient au fait que l'ambidextérité apparaît comme un mécanisme permettant à une entreprise de faire face efficacement aux défis de la concurrence et aux chocs environnementaux pour assurer sa survie et son succès (AtuaheneGima, 2005). Aussi, la compréhension du rôle de l'ambidextérité, en période de crise pandémique, dans un pays en développement comme la République du Congo permet d'étendre la théorie actuelle à de nouveaux contextes.

Le deuxième commentaire est que ce travail contribue à la littérature en montrant que l'ambidextérité organisationnelle n'est pas limitée à un niveau organisationnel spécifique. Plus précisément, il explore l'ambidextérité à travers les niveaux d'activités des femmes et soutient que les interactions synchronisées entre l'exploration et l'exploitation permettent aux activités développées par les femmes de devenir ambidextres (voir également Cantarello Nosella et Filippini, 2012) et de faire face à la crise pandémique. 
Enfin, le troisième commentaire émane du fait que l'ambidextérité ne se limite pas aux produits existants. De ce fait, la contribution de ce travail repose sur le fait que la survie et le succès des activités entrepreneuriales des femmes reposent sur leur capacité à innover de nouveaux produits et à renforcer simultanément la performance continue de produits établis (Christensen, 1997; Tripsas, 1997; Smith et Tushman, 2005). Par conséquent, l'ambidextérité peut être le moteur de la survie et du succès des activités des femmes entrepreneures lorsqu'elle permet de synchroniser le développement et la commercialisation de produits nouveaux et établis (Smith et Tushman, 2005).

\section{Méthodologie}

Cette section est structurée en deux points. Le premier est consacré à la méthodologie de collecte des récits de vie tandis que le second porte sur la présentation des récits de vie des femmes promotrices de microentreprises en pleine crise pandémique du coronavirus.

\subsection{Collecte des récits de vie}

La méthodologie utilisée pour analyser les effets de la pandémie du coronavirus sur les microentreprises des femmes est celle des récits de vie. Elle a la particularité de mettre l'accent sur les personnes, notamment les aspects particuliers de l'expérience vécue (p. ex., un choc (variation des prix) affectant les intrants ou une baisse de la demande dans l'exercice d'une activité). Cette méthode, qui permet de créer une connaissance narrative au sens de Bruner (1986), prend appui sur le récit fait par une personne au sujet de son expérience vécue et de l'importance qu'elle accorde à cette expérience, au fil du temps ou à un moment donné, et qui peut évoluer au fur et à mesure du déroulement de l'histoire. Ce type de construction du savoir nous invite à prêter attention aux détails des récits et au contexte dans lequel ils s'inscrivent.

Ainsi, le recours aux récits de vie pour éclairer notre compréhension des effets de la crise pandémique $\mathrm{du}$ coronavirus sur les microentreprises des femmes est particulièrement utile pour plusieurs raisons. D'abord, les récits de vie ont été recueillis pendant que Brazzaville est en confinement, ce qui facilite le recueil de récits des femmes qui sont épargnées d'un effort de rappel. Ensuite, ils ont l'avantage de relier les aspects macroéconomiques et microéconomiques parce qu'ils permettent aux individus de parler d'eux-mêmes et de leur vie, mais aussi de leurs environnements socioéconomique et politique. Aussi, les récits de vie peuvent être utilisés pour communiquer sur la manière dont la microentreprise d'une femme et son environnement se recoupent pour produire des circonstances de la vie des femmes.

Enfin, les récits de vie permettent de comprendre les processus de changement. Ils peuvent être utilisés pour cartographier la trajectoire d'une microentreprise et pour identifier les ressorts de ce changement. Dans ce sens, les récits de vie peuvent éclairer les connaissances préexistantes sur les microentreprises féminines, refléter les idées fausses courantes et préjudiciables ainsi que générer des résultats contre-intuitifs, ce qui stimule de nouveaux domaines de recherche.

Pour cette recherche, nous avons retenu six récits de vie recueillis dans la période allant du 2 au 30 avril 2020. Cette période est celle du confinement de la population avec l'arrêt des transports publics, le couvre-feu de 20 h à 5 h du matin, l'arrêt de toutes les activités non essentielles et la réduction du nombre de jours d'ouverture des marchés domaniaux (lundi, mercredi et vendredi). Les six récits recueillis concernent les activités agricoles, les activités commerciales et les activités artisanales. Le choix porté sur ces secteurs est dû à leur prépondérance ${ }^{2}$ dans les activités microentrepreneuriales des femmes.

\subsection{Récits de vie des femmes en période de crise pandémique}

Les six récits de vie recueillis pendant la période du confinement ne constituent pas un échantillon représentatif. Ils permettent toutefois d'illustrer l'existence d'une dynamique dans une société, précisément chez les femmes engagées dans des activités génératrices de revenus dans les secteurs agricoles, du commerce et de l'artisanat. Pour des raisons de concision, seuls trois récits sont présentés dans le corps du texte; les trois autres sont placés en annexe. Voici donc les récits de mesdames Julie, Justine et Aubierge. 


\subsubsection{Récit de madame Julie : une fonctionnaire engagée dans les activités agropastorales}

«Je m’appelle Julie. Je suis fonctionnaire et j'exerce des activités agro-pastorales à Brazzaville (Manianga, Djiri et environs) et au point kilométrique 45. Dans le cadre de l'agriculture, je fais l'arboriculture, le maraichage et les cultures associées du manioc et divers (piment, oseille, etc). En matière d'élevage, je fais de la pisciculture, les petits ruminants et la volaille (canards, oies, poules pondeuses et poules locales/hybrides).

La crise sanitaire a énormément modifié ma présence en tant que fonctionnaire sur le lieu d'exercice de mon activité. Avant la crise, je ne consacrais que peu de temps dans la journée à mon activité : environ $2 \mathrm{~h}$ par jour les jours ouvrables et à plein temps le week-end et les jours fériés. Mais, avec la crise, j’y consacre plus de temps. Les jours de plein régime sont ceux d'ouverture des marchés (lundi, mercredi et vendredi). Les autres jours sont partagés entre les restrictions dues au coronavirus et le suivi de l'activité. Si les activités de Manianga (proche de la maison) sont visitées tous les jours, celles du PK 45 le sont moins; deux fois, depuis le 31 mars 2020, tandis qu'elles l'étaient à la fréquence d'au moins deux jours dans la semaine.

La pratique de notre activité a été également impactée par cette crise. Pour preuve, avant la crise, nous produisions une partie de nos semences et en achetions une autre partie dans les maisons de distribution de la ville (viaduc, Texaco, marché Total, etc). Mais, depuis qu'il y a la crise, nous avons arrêté de produire les semences. Aussi, la forte pluviométrie aggrave la situation : les fortes pluies ont détruit les cultures et inondé les espaces de cultures. Pour faire face aux pertes occasionnées par les pluies et par la baisse des clients en période de crise pandémique, nous avons vendu le reste de la production à vil prix. Il faut aussi dire que nous ne nous approvisionnons plus régulièrement. Du fait des risques de contagion, nous avons limité nos déplacements entre le viaduc et Texaco pour les approvisionnements en intrants agricoles.

Pour la conduite de notre activité, nous utilisons une équipe de six employés permanents et de trois «mercenaires » ou tâcherons par semaine pour les deux sites de production de Brazzaville. Avec la crise, nous avons plusieurs sollicitations, notamment de la part des jeunes hommes, ayant pour les uns perdu leur emploi du fait de la crise et pour les autres étant en congé technique du fait des inondations. Même une catégorie d'anciens propriétaires/patrons de sites/planches inondées sont venus rechercher une bouffée financière pour la relance de leurs activités. Nous avons sacrifié nos marges bénéficiaires, car la crise recommande une assistance sociale de la part de tous. C'est pourquoi nous nous sommes résolus à ne laisser personne en marge.

Avant la crise, nous avions un réseau de clients et la livraison se faisait à domicile et dans les marchés, notamment ceux de Texaco et Massengo. Avec la limitation des mouvements due à la pandémie de coronavirus, nous avons développé de nouveaux circuits de livraison aux alentours des sites de production, à la conquête des zones d'accès difficiles telles que Trois-Poteaux à Massengo. Nous procédons ainsi pour atteindre certaines commerçantes qui n'ont pas de moyen de transport et qui ne peuvent pas alimenter les marchés.

Nous disposons d'un véhicule (berline) qui a été mis en contribution pour les déplacements du personnel. Avant la crise, l'ensemble du personnel empruntait les transports en commun. Depuis la crise, le personnel tant permanent que temporaire arrive aux sites en empruntant les occasions qui s'offrent à chacun. Pour le retour, le véhicule réduit les distances à parcourir en déposant les employés à certains points précis.

Pour la sécurité sanitaire des employés, nous exigions le respect des gestes barrière, notamment la distanciation physique. Le lavage des mains au savon est respecté, mais pas l'usage du gel hydroalcoolique, car il n'est pas à la portée de tous (onéreux). Toutefois, il convient de relever l'incrédulité de certains employés, qui estiment que l'exposition au soleil des maraichers et les activités physiques intenses réduisent le risque de contamination des Africains, et ce, paradoxalement au nombre d'africains fauchés par la maladie aux États Unis.

Certains n’hésitent pas à dire tout haut que le coronavirus, c'est pour eux, les « Blancs », que « Dieu est notre gardien » ou encore que « Dieu les punit parce qu'ils ont osé le braver en autorisant les mariages de même sexe ». Pour une catégorie de personnes analphabètes et semi-analphabètes, nous essayons d'adapter les messages de sensibilisation à leur 
niveau et de leur présenter les statistiques mondiales, sans oublier de leur présenter des illustrations. Cependant, si les doutes dans les esprits perdurent, c'est surtout du fait que jusqu'à ce jour, à leur entendement, aucun cas nommé n'a été présenté à la télévision et aucun voisin ni aucune connaissance n'a perdu un proche de la maladie.

L'apport de ma famille est essentiel dans l'exercice de cette activité. Mon conjoint m'a toujours soutenue moralement en m'encourageant et en m'apportant un appui financier. Mes enfants m'aidaient aux sites avant la crise. Mais, pour les protéger, il leur est interdit d'y aller. Ils restent, néanmoins, attentifs à ma personne au retour de l'activité. Ils veillent à ce que je lave mes mains, prenne une douche et demandent le récit du vécu des histoires hors maison. Ils sont admiratifs des images de production et se pressent de les publier sur la Toile. »

\subsubsection{Récit de madame Justine : une femme engagée dans une activité de cuisine et vente de beignets}

«Je suis madame Justine. Je suis une femme célibataire avec six enfants à charge; des orphelins de ma sœur ainée. Pour vivre, j'exerce l'activité de fabrication et de vente de beignets. Je pratique cette activité depuis une quinzaine d'années. La pandémie de coronavirus a induit beaucoup de changements dans l'exercice de mon activité. Avant la crise, je m'approvisionnais en intrants sans problème. Maintenant, mon approvisionnement en intrants devient très difficile. Par exemple, les déplacements sont difficiles. Il y a des problèmes d'eau et les prix des intrants ont augmenté. Le transporteur (koro-koro) que j'empruntais pour transporter mes achats du marché à la maison me demande, aujourd'hui 1500 francs CFA (3\$) contre 500 francs CFA (1\$), en temps normal.

L'exercice de mon activité a été fortement impacté par cette crise. Avant, je faisais ma pâte à 15 h et je commençais la fabrication et la vente des beignets à 17 h, pour terminer à 22 h, parfois 23 h. Maintenant, je dois commencer la fabrication et la vente des

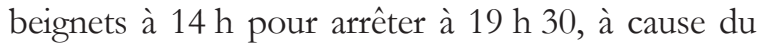
couvre-feu qui débute à 20 h. En dehors de ça, j’ai peur de la contamination. Lorsque les clients arrivent, je leur fais obligation de respecter les gestes barrière, notamment la distanciation physique d'un mètre et le port du masque.
Les ventes ont baissé. L'activité ne fonctionne plus comme avant. En temps normal, je vendais pour 25000 francs CFA la journée. Maintenant, j’atteins à peine 15000 francs CFA. La crise de coronavirus a changé beaucoup de choses. Quand je pouvais travailler jusqu'à $22 \mathrm{~h}$, j'avais tout le temps pour faire mon travail et m'occuper des enfants. Avec la crise, je suis pressée par le temps. Il m'arrive d'abandonner mon travail (tâches domestiques) pour aller vite préparer la pâte des beignets. Mon emploi du temps a beaucoup changé. Les jours de marché (lundi, mercredi et vendredi), je fais très tôt les tâches domestiques. Après, je dois aller rapidement au marché pour m'approvisionner en intrants et en produits alimentaires pour le repas de la famille.

Le temps est très court. Je n'ai personne pour m'aider. J'ai des orphelins à élever et je le fais toute seule. Les enfants ne peuvent pas sortir de la maison à cause du coronavirus. Avant, les enfants m'aidaient et allégeaient mes tâches. Avec cette crise, je ne voudrais pas leur faire prendre des risques. Vous savez, les enfants peuvent ou ne pas se laver les mains. »

\subsubsection{Récit de madame Aubierge : une femme couturière}

"Je suis madame Aubierge et je suis couturière. J'exerce cette activité depuis quatre ans. Avant la crise sanitaire, les gens m'apportaient leurs tissus, je confectionnais des habits pour eux et tout allait bien. Il m'arrivait aussi d'acheter mes propres tissus pour coudre des tenues qui étaient bien vendues. Depuis qu'il y a la crise, les gens n'achètent plus. Ils ont peutêtre peur de la maladie. En tout cas, l'activité ne marche plus et, à un moment donné, j'avais baissé les bras et je ne faisais que de petites retouches pour les habitants du quartier qui me contactaient à domicile.

Depuis que le gouvernement a demandé aux couturiers de travailler pour fabriquer des masques artisanaux, j’ai repris de l'activité. Je produis des masques que je fais vendre par mon fils dans les arrêts de bus et les grandes artères de la ville. Mon fils arrive à écouler facilement la production et, après quelques heures, il revient pour me dire que "Maman, c'est fini".

Avant la crise sanitaire, j'allais moi-même au marché et à la mercerie pour m'approvisionner en intrants. Maintenant, ce sont les fournisseurs qui nous font la livraison à domicile. Je les contacte au téléphone pour passer ma commande et, au moyen d'une 
moto, le fournisseur me la livre à mon lieu de travail parce qu'il n'y a pas les transports en commun. Cette activité me met en contact avec des gens et il me faut prendre des précautions pour ne pas être contaminée. Je me protège avec un masque et je me lave les mains régulièrement. En plus, j’ai des gants.

Mon activité me permet de vivre. C'est vrai que la grande activité n'y est plus, mais, en ce qui concerne la production des masques, Dieu est en train de faire $^{3}$. Avant la crise, la couture rapportait beaucoup. Maintenant, la production des masques artisanaux me permet juste de prendre en charge ma famille. Avant, je pouvais faire des économies pour acheter mes propres tissus et produire pour la vente. Maintenant, ce n'est plus possible. Tout va dans la nourriture. La crise sanitaire a changé aussi mes jours de travail. Avant le confinement, je travaillais du mardi au samedi. Je ne travaillais pas les dimanches et lundis. Depuis le confinement, je travaille seulement les jours du marché (lundi, mercredi et vendredi). Il y a des moments où les gens du quartier m'apportent les tissus à la maison pour que je confectionne des habits pour eux. Les jours ouvrables, je travaille de $8 \mathrm{~h}$ à $17 \mathrm{~h}$, avant comme pendant la crise. Il faut tout de même noter qu'en temps normal, le mardi, je finis à $15 \mathrm{~h}$ pour aller à la prière.

Dans l'exercice de mon travail, je bénéficie de l'aide de deux apprenties. Actuellement, elles ne sont pas disponibles parce qu'il y en a une qui est enceinte et l'autre en voyage. Je n'ai personne, en ce moment. Ainsi, lorsque j'ai une course, je suis obligée de fermer boutique. Je me bats seulement comme ça. Une fois, nous avons été contactés par les gens du ministère responsable des PME et de l'artisanat pour la production des masques. Il était question qu'ils nous rappellent, mais il n'y a pas eu de suite jusqu'à aujourd'hui.

J'ai commencé comme vendeuse au marché de Bouemba. Lorsque j'ai eu un peu d'argent, j'ai décidé toute seule d'aller apprendre la couture. Je n'ai jamais bénéficié d'une aide quelconque du gouvernement, alors que j'en ai besoin pour développer mon activité. Depuis le début de la crise, j’aide les gens de mon quartier à se protéger du coronavirus. Avec les restes des tissus, je fabrique des masques, que je distribue gratuitement à mes voisins. »

\section{Discussion}

Dans un contexte d'incertitude engendrée par la crise de la COVID-19, les agents économiques ont un comportement de minimisation de risque (Hugon, 1996). Pour souligner ce fait, Haubert (1999) relève que plus les conditions de reproduction sont précaires, plus le comportement de la personne qui développe l'activité familiale est marqué par l'aversion pour le risque. Les stratégies de diversification, de recherche de nouveaux marchés et de flexibilité de la main-d'œuvre peuvent constituer un moyen de minimiser le risque. Aussi, cette discussion va-t-elle porter sur l'identification des stratégies déployées par les femmes entrepreneures et sur le caractère innovateur de ces stratégies.

\subsection{Identification des stratégies développées par les entrepreneures}

La première préoccupation dans l'analyse est d'identifier les stratégies développées par les femmes propriétaires de microentreprises en pleine crise de pandémie. Pour ce faire, il est indispensable de croiser les réactions de ces entrepreneures aux différentes mesures ayant caractérisé la crise pandémique du coronavirus en distinguant les femmes évoluant dans le domaine agricole de celles évoluant dans le commerce et l'artisanat.

\subsubsection{Stratégies dans le secteur agricole}

Dans le secteur agricole, nous nous servons du récit de madame Julie qui tient des activités agropastorales et de celui de madame Raguie qui est maraichère. Les deux récits permettent d'identifier les stratégies mises en place par ces deux entrepreneures pour continuer à faire vivre leurs activités en pleine crise pandémique, comme le montre le tableau suivant: 


\begin{tabular}{|c|c|c|}
\hline \multirow{2}{*}{$\begin{array}{l}\text { Mesures de lutte } \\
\text { contre le coronavirus }\end{array}$} & \multicolumn{2}{|c|}{ Réaction } \\
\hline & Madame Julie (ferme agropastorale) & Madame Raguie (maraichère) \\
\hline $\begin{array}{l}\text { Arrêt des transports } \\
\text { en commun }\end{array}$ & $\begin{array}{l}\text { Dispose d'une voiture et aide son } \\
\text { personnel pour se déplacer en fin de } \\
\text { journée. }\end{array}$ & $\begin{array}{l}\text { A opté pour le déplacement à pied à } \\
\text { raison de } 3 \mathrm{~h} \text { le matin (pour l'aller) et } \\
3 \mathrm{~h} \text { l'après-midi (pour le retour). }\end{array}$ \\
\hline $\begin{array}{l}\text { Confinement de la } \\
\text { main-d'œuvre }\end{array}$ & $\begin{array}{l}\text { A employé les personnes qui se sont } \\
\text { retrouvées au chômage dans son quartier. }\end{array}$ & $\begin{array}{l}\text { Ne change rien, car elle a toujours } \\
\text { travaillé seule avec l'aide de ses enfants. }\end{array}$ \\
\hline $\begin{array}{l}\text { Réduction du } \\
\text { nombre de jours } \\
\text { ouvrables des } \\
\text { marchés (lundi, } \\
\text { mercredi et vendredi) }\end{array}$ & $\begin{array}{l}\text { En dehors des } 3 \text { jours de marché, elle fait } \\
\text { la livraison à domicile et recherche des } \\
\text { clients dans de nouveaux quartiers } \\
\text { proches de son lieu d'habitation; a aussi } \\
\text { baissé les prix de ses produits. }\end{array}$ & $\begin{array}{l}\text { En dehors des } 3 \text { jours de marché, elle } \\
\text { vend tout le long de son chemin de } \\
\text { retour à la maison. }\end{array}$ \\
\hline $\begin{array}{l}\text { Interdiction de toutes } \\
\text { les activités non } \\
\text { essentielles }\end{array}$ & $\begin{array}{l}\text { Pas de problème, car c'est une activité } \\
\text { essentielle pour la vie des populations. }\end{array}$ & $\begin{array}{l}\text { Pas de problème, car c'est une activité } \\
\text { essentielle pour la vie des populations. }\end{array}$ \\
\hline $\begin{array}{l}\text { Couvre-feu (de } 20 \text { h à } \\
5 \text { h) }\end{array}$ & $\begin{array}{l}\text { Le temps de travail reste le même, mais a } \\
\text { réduit la fréquence de déplacement pour } \\
\text { les sites à l'extérieur de Brazzaville. }\end{array}$ & $\begin{array}{l}\text { A réduit le temps de travail de } 10 \text { h à } \\
15 \text { h, au lieu de } 8 \text { h à } 16 \text { h }\end{array}$ \\
\hline
\end{tabular}

Tableau 1 - Réaction des femmes entrepreneures dans

le secteur agricole en période de crise pandémique

Source : L'auteur, à partir des récits de vie de mesdames Julie et Raguie

La lecture de ce tableau permet de soutenir l'idée selon laquelle les stratégies développées par les deux femmes qui évoluent dans le domaine agricole relèvent de l'ambidextrie. Ce concept développé par Duncan (1976) et March (1991) met l'accent sur l'exploitation et l'exploration non seulement dans les processus d'apprentissage, comme l'ont montré ces deux auteurs, mais aussi dans les processus d'adaptation en période de crise, comme il en est question ici. L'exploitation renvoie au raffinement, à l'efficacité, à la sélection et à la mise en œuvre. L'exploration, quant à elle, réfère à la recherche, à la variation, à l'expérimentation et à l'innovation (Koubaa, 2017; ÚbedaGarcía, Claver-Cortés, Marco-Lajara et ZaragozaSáez, 2017).

\section{Stratégies d'exploitation}

En ce qui concerne l'exploitation, les deux femmes ont poursuivi leurs activités, en dépit des mesures prises pour lutter contre la pandémie de coronavirus. Les deux récits permettent de faire le constat selon lequel les femmes évoluant dans l'agriculture n'ont pas subi toutes les mesures liées au coronavirus. C'est le cas de la mesure de l'interdiction de toutes les activités non essentielles; les activités agropastorales étant considérées comme étant indispensables à la survie de l'humain. Dès lors, la possibilité leur était donnée de poursuivre leurs activités, même au moment le plus difficile de la crise.

Seulement, la poursuite de l'exploitation de la microentreprise des deux femmes permet de faire un second constat relatif à la différence de réaction entre les activités n'ayant pas la même dimension ni la même organisation. Ainsi, madame Julie, qui a une plus grande exploitation et plus de moyens a opté pour une stratégie de ressources. Par contre, madame Raguie, qui a une petite exploitation de produits maraichers et moins de moyens, a opté pour une stratégie de repli. En effet, madame Julie a mobilisé sa voiture pour le transport de son personnel afin de répondre à l'arrêt des transports en commun, tandis que que madame Raguie s'est imposé une marche de $6 \mathrm{~h}$ par jour. Pour assurer la continuité de leurs activités, madame Julie a embauché du personnel (des voisins qui ont perdu leur emploi en raison de la crise), tandis que que madame Raguie a continué à travailler toute seule.

\section{Stratégies d'exploration}

Au sujet de l'exploration, il est à noter qu'en dépit des différences, les deux entrepreneures ont un point commun. Il s'agit du fait que, devant le petit nombre 
de jours de marché, madame Julie explore de nouveaux clients dans des quartiers qu'elle n'avait pas explorés avant la crise. Madame Raguie, quant à elle, vend une partie de ses planches de légumes aux vendeuses des marchés et le reste est vendu sur son chemin de retour en marchant.

\subsubsection{Stratégies dans les secteur du commerce et de l'artisanat}

Dans les secteurs du commerce et de l'artisanat, nous avons recueilli le récit de trois femmes: 1) une vendeuse de pain, 2) une cuisinière et vendeuse de beignets et 3) une couturière. Ces récits permettent d'identifier les stratégies mises en place par ces trois entrepreneures pour continuer leurs activités en pleine crise pandémique coronavirus, comme le montre le tableau suivant :

\begin{tabular}{|c|c|c|c|}
\hline \multirow{2}{*}{$\begin{array}{l}\text { Mesures de } \\
\text { lutte contre le } \\
\text { coronavirus }\end{array}$} & \multicolumn{3}{|c|}{ Réaction } \\
\hline & $\begin{array}{c}\text { Madame Inès } \\
\text { (vendeuse de pain) }\end{array}$ & $\begin{array}{c}\text { Madame Justine } \\
\text { (vendeuse de beignets) }\end{array}$ & $\begin{array}{l}\text { Madame Aubierge } \\
\text { (couturière) }\end{array}$ \\
\hline $\begin{array}{l}\text { Arrêt des } \\
\text { transports en } \\
\text { commun }\end{array}$ & $\begin{array}{l}\text { Elle n'a pas un problème } \\
\text { de déplacement, car la } \\
\text { boulangerie livre des } \\
\text { pains à la boutique, qui } \\
\text { est dans le quartier. }\end{array}$ & $\begin{array}{l}\text { Elle se déplace à pied. } \\
\text { Pour le transport des } \\
\text { intrants, elle a recours } \\
\text { aux transporteurs par la } \\
\text { brouette (koro-koro). }\end{array}$ & $\begin{array}{l}\text { Elle n'a pas de } \\
\text { problème de } \\
\text { déplacement, car les } \\
\text { fournisseurs lui font la } \\
\text { livraison à domicile. }\end{array}$ \\
\hline $\begin{array}{l}\text { Confinement de } \\
\text { la main-d'œuvre }\end{array}$ & $\begin{array}{l}\text { Ses deux employés étant } \\
\text { confinés, elle se fait aider } \\
\text { par son frère. }\end{array}$ & $\begin{array}{l}\text { Pas de problème, car elle } \\
\text { travaille seule et se fait } \\
\text { parfois aider par les } \\
\text { enfants. }\end{array}$ & $\begin{array}{l}\text { Pas de problème, car } \\
\text { elle travaille seule, } \\
\text { même avant la crise. }\end{array}$ \\
\hline $\begin{array}{l}\text { Réduction du } \\
\text { nombre de jours } \\
\text { ouvrables des } \\
\text { marchés (lundi, } \\
\text { mercredi et } \\
\text { vendredi) }\end{array}$ & $\begin{array}{l}\text { Pas de problème, car son } \\
\text { activité porte sur un } \\
\text { produit alimentaire } \\
\text { autorisé à être vendu } \\
\text { tous les jours. }\end{array}$ & $\begin{array}{l}\text { Elle s'est adaptée en } \\
\text { réduisant le nombre de } \\
\text { jours d'exercice de ses } \\
\text { activités aux jours } \\
\text { ouvrables des marchés. }\end{array}$ & $\begin{array}{l}\text { Elle s'est adaptée en } \\
\text { réduisant le nombre de } \\
\text { jours d'exercice de ses } \\
\text { activités aux jours } \\
\text { ouvrables des marchés. }\end{array}$ \\
\hline $\begin{array}{l}\text { Interdiction de } \\
\text { toutes les } \\
\text { activités non } \\
\text { essentielles }\end{array}$ & $\begin{array}{l}\text { Elle n'a pas changé } \\
\text { d'activité ni développé } \\
\text { une autre, car la crise a } \\
\text { été une opportunité pour } \\
\text { développer son activité, } \\
\text { qui était jusqu'ici } \\
\text { méconnue. }\end{array}$ & $\begin{array}{l}\text { Malgré la baisse des } \\
\text { revenus, elle a continué } \\
\text { dans la même activité } \\
\text { pour avoir ne serait-ce } \\
\text { que de quoi survivre. }\end{array}$ & $\begin{array}{l}\text { Elle a réorienté son } \\
\text { activité en produisant } \\
\text { des masques artisanaux. }\end{array}$ \\
\hline $\begin{array}{l}\text { Couvre-feu (de } \\
20 \text { h à } 5 \text { h) }\end{array}$ & $\begin{array}{l}\text { En temps normal, elle } \\
\text { travaille de } 6 \text { h à } 22 \text { h. } \\
\text { Avec la crise, c'est de } 8 \text { h } \\
\text { à } 19 \text { h } 30 .\end{array}$ & $\begin{array}{l}\text { Avant la crise : } 17 \text { h à } \\
22 \text { h, parfois } 23 \text { h. } \\
\text { Avec la crise, de } 14 \text { h } 30 \\
\text { à } 19 \text { h } 30 .\end{array}$ & $\begin{array}{l}\text { Elle n’a pas changé ses } \\
\text { horaires de travail : } \\
\text { toujours de } 8 \text { h à } 17 \text { h. }\end{array}$ \\
\hline
\end{tabular}

Tableau 2 - Réaction des femmes entrepreneures dans les secteurs

du commerce et de l'artisanat en période de crise pandémique Source : L'auteur, à partir des récits de vie de mesdames Inès, Justine et Aubierge

La lecture du tableau ci-dessus relatif aux stratégies d'adaptation d'entrepreneures évoluant dans le commerce et l'artisanat permet d'identifier trois types de stratégies leur ayant permis de faire face à la crise pandémique :

\section{1) Le développement et l'introduction d'un nowveau produit:}

Il s'agit du cas de madame Aubierge. Habituée à réaliser son chiffre d'affaires grâce à la confection d'uniformes pour les mariages et les cérémonies de décès, elle a dû se résoudre à produire des masques artisanaux pour s'adapter à la crise; 


\section{2) La flexibilité dans la gestion de la main-d'cuvre:}

À ce sujet, les récits révèlent que les femmes ont souvent recours à la main-d'œuvre familiale, à l'instar de madame Inès, vendeuse de pain qui fait appel à son frère en raison du confinement de ses deux vendeuses. Il en est de même de mesdames Justine et Aubierge qui font appel à leurs enfants.

\section{3) L'ajustement des jours et des heures de travail:}

Mesdames Inès et Justine ont ajusté leur horaire de travail en fonction des heures du couvre-feu. C'est pourquoi ces dernières ont décidé d'arrêter le travail à 19 h 30, alors qu'avant la crise, elles travaillaient jusqu'à $22 \mathrm{~h}$, voire au-delà.

Les stratégies développées par ces femmes sont en accord avec la littérature relative à la capacité d'adaptation des microentreprises en général et des entreprises féminines en particulier. Ces microentreprises sont souvent très flexibles et, de ce fait, disposent d'une forte capacité d'adaptation aux chocs affectant l'environnement économique. Plus aptes à exploiter des niches de marché (Narjoko et Hill, 2007; Hodorogel, 2009), les microentreprises se concentrent souvent sur des activités caractérisées par des économies d'agglomération, plutôt que par des économies d'échelle (Berry, Rodriguez et Sande, 2001; Hall et Harvie, 2003), et dépendent moins des crédits formels que les autres entreprises (PME, petites et moyennes industries, grandes entreprises), qui sont plus accablées par des dettes (Sato, 2000; ter Wengel et Rodriguez, 2006). Même ce qui représente leur talon d'Achille, à savoir le faible niveau technologique et des connaissances peut être surmonté en imitant les meilleures pratiques d'autres entreprises (Nugent et Yhee, 2002). Ainsi, les microentreprises féminines peuvent être plus à même de maintenir leur viabilité et, donc, de contrer les effets négatifs de la crise.
Dans les trois cas présentés des secteurs du commerce et de l'artisanat, il est possible de soutenir l'existence de l'ambidextrie par le fait que l'activité d'exploitation s'est poursuivie durant la crise dans la mesure où le pain, les beignets et les masques artisanaux sont essentiels pour la survie des populations. L'activité d'exploration s'est faite aussi pendant cette période à travers la recherche de clients pour les masques artisanaux et de clients de proximité pour les beignets dans le quartier. Pendant le confinement, le schéma du client qui se rapproche du point de vente pour s'approvisionner a été dans certains cas modifié par celui du vendeur qui a laissé son point de vente habituel pour aller à la recherche du client en passant de ménage en ménage. Ainsi, pendant la crise du coronavirus, l'exploration de niches de clients s'est développée.

\subsection{Stratégies d'adaptation ou innovations sociales}

L'analyse des stratégies développées par les femmes en période de confinement, dans les récits de vie étudiés, montre que trois stratégies ont été particulièrement utilisées : 1) la diversification des produits (production de masques artisanaux), 2) L'exploration de nouvelles niches de clients (vente de produits agricoles dans les quartiers) et 3) la flexibilité dans la gestion de la main-d'œuvre (recours à l'appui des membres de la famille pour faire fonctionner les activités). Or, peut-on considérer ces stratégies comme des innovations sociales? Pour répondre à cette question, les trois stratégies nécessitent d'être mises en lumière par rapport aux critères de Bund et ses collègues (2013) évoqués dans l'introduction. Le tableau ci-dessous présente cette analyse : 


\begin{tabular}{|c|c|c|c|}
\hline Stratégies/Critères & $\begin{array}{l}\text { Diversification des } \\
\text { produits }\end{array}$ & $\begin{array}{l}\text { Exploration de nouvelles } \\
\text { niches de clients }\end{array}$ & $\begin{array}{c}\text { Flexibilité dans la } \\
\text { gestion de la main- } \\
\text { d'œuvre }\end{array}$ \\
\hline Exemple & $\begin{array}{c}\text { Production de masques } \\
\text { artisanaux }\end{array}$ & $\begin{array}{c}\text { Vente de produits } \\
\text { agricoles dans les } \\
\text { quartiers }\end{array}$ & $\begin{array}{l}\text { Recours à l'appui des } \\
\text { membres de la famille }\end{array}$ \\
\hline $\begin{array}{l}\text { Satisfait les besoins } \\
\text { sociaux en matière } \\
\text { d'emploi, de santé, } \\
\text { d'éducation, etc. }\end{array}$ & $\begin{array}{c}\text { Constitue une } \\
\text { protection essentielle } \\
\text { dans la lutte contre la } \\
\text { COVID-19. }\end{array}$ & $\begin{array}{l}\text { Permet d'apporter aux } \\
\text { ménages les produits } \\
\text { agricoles jusqu'ici vendus } \\
\text { dans les marchés ou à des } \\
\text { endroits bien précis. }\end{array}$ & $\begin{array}{l}\text { Permet de baisser les } \\
\text { coûts et de dégager des } \\
\text { revenus pour l'entretien } \\
\text { de la famille. }\end{array}$ \\
\hline $\begin{array}{l}\text { Comporte des } \\
\text { éléments } \\
\text { nouveaux (du } \\
\text { moins dans leur } \\
\text { contexte) }\end{array}$ & $\begin{array}{l}\text { Les masques artisanaux } \\
\text { ont été développés pour } \\
\text { pallier l'insuffisance des } \\
\text { masques chirurgicaux } \\
\text { importés et plus } \\
\text { coûteux. }\end{array}$ & $\begin{array}{l}\text { Propose les produits } \\
\text { maraichers aux domiciles } \\
\text { des ménages (crieurs) et } \\
\text { dans les quartiers non } \\
\text { explorés jusqu'ici. }\end{array}$ & $\begin{array}{l}\text { Les membres de la famille } \\
\text { ont toujours été, au } \\
\text { minimum, une solution } \\
\text { d'appoint au problème de } \\
\text { la main-d'œuvre dans les } \\
\text { microentreprises. }\end{array}$ \\
\hline $\begin{array}{l}\text { Effectivement } \\
\text { mise en œuvre (est } \\
\text { loin d'être une } \\
\text { simple idée) }\end{array}$ & $\begin{array}{l}\text { Cette stratégie est } \\
\text { devenue une activité- } \\
\text { phare des couturiers } \\
\text { pendant la crise. }\end{array}$ & $\begin{array}{l}\text { Cette stratégie est utilisée } \\
\text { aussi bien par les } \\
\text { maraichères que par les } \\
\text { vendeuses de beignets. }\end{array}$ & $\begin{array}{c}\text { Cette solution est } \\
\text { beaucoup utilisée en } \\
\text { temps normal et encore } \\
\text { plus en période de crise, } \\
\text { notamment de } \\
\text { confinement. }\end{array}$ \\
\hline $\begin{array}{l}\text { Fonctionne bien } \\
\text { (est plus efficace } \\
\text { que les solutions } \\
\text { traditionnelles) }\end{array}$ & $\begin{array}{l}\text { Les masques artisanaux } \\
\text { sont aussi efficaces que } \\
\text { les masques importés et } \\
\text { sont à moindre coût. }\end{array}$ & $\begin{array}{l}\text { Cette stratégie a bien } \\
\text { fonctionné, surtout en } \\
\text { période de confinement. }\end{array}$ & $\begin{array}{c}\text { Cette solution fonctionne } \\
\text { bien, surtout en période } \\
\text { de crise. }\end{array}$ \\
\hline Conclusion & $\begin{array}{l}\text { Cette stratégie est } \\
\text { nouvelle et satisfait à } \\
\text { tous les critères. Elle } \\
\text { peut être considérée } \\
\text { comme une innovation } \\
\text { sociale. }\end{array}$ & $\begin{array}{l}\text { Bien qu'étant une extension } \\
\text { de ce qui est déjà observé } \\
\text { dans la cordonnerie } \\
\text { artisanale, cette stratégie } \\
\text { peut être considérée comme } \\
\text { une innovation sociale. }\end{array}$ & $\begin{array}{l}\text { Il s'agit d'une stratégie } \\
\text { traditionnelle qui a pris de } \\
\text { l'ampleur en période } \\
\text { de crise. }\end{array}$ \\
\hline
\end{tabular}

Tableau 3 - Critères de Bund et ses collègues (2013) et stratégies développées par les femmes entrepreneures Source : L'auteur

La lecture de ce tableau suggère que, dans la pratique, des femmes entrepreneures développent en période de crise des stratégies dont certaines peuvent être classées comme des innovations sociales, alors que d'autres demeurent de simples stratégies d'adaptation.

En ce qui concerne les stratégies pouvant être considérées comme des innovations sociales, le tableau 3 permet d'en identifier deux, à savoir la production de masques artisanaux (diversification de produits) et la vente ambulante de produits maraichers (exploration de nouvelles niches de clients). Ces deux stratégies satisfont à tous les critères de Bund et ses collègues (2013). Elles permettent de satisfaire des besoins sociaux, en l'occurrence le besoin de nutrition et de préservation de l'état de santé des populations confinées en raison de la crise. De plus, elles comportent des éléments nouveaux, dans la mesure où la production de masques artisanaux est une nouveauté dans l'activité de couture. En revanche, la vente ambulante de produits maraichers pour atteindre les clients à leur domicile n'a rien de nouveau à Brazzaville (Missié, 2005) et dans les villes africaines (Monnet, 2006).

\section{Exploration de nouvelles niches de clients}

En effet, dans les villes africaines, il est courant de voir les vendeurs de fruits le long des avenues et des rues pour vendre leurs produits. Il en est de même des cordonniers ambulants, qui proposent leurs services aux domiciles des clients. Ce qui est nouveau est l'extension de cette pratique aux produits maraichers qui, jusqu'ici, n'étaient vendus que dans les marchés ou les hangars qui offrent les meilleures conditions de 
conservation en matière de protection contre le soleil et la facilité d'accès à l'eau pour rafraichir les produits. Ainsi, la vente ambulante des produits maraichers dans une cuvette avec de l'eau, tout en évitant une exposition prolongée au soleil, est la nouveauté dans cette stratégie développée par les femmes.

\section{Diversification des produits}

Il convient de relever que, pour une microentreprise aux prises avec des aléas environnementaux, le développement et l'introduction de nouveaux produits sont essentiels à sa survie. Cooper et McIntyre (1993) considère cette stratégie comme étant la plus risquée. Pourtant, elle est la plus importante pour une entreprise en présence de situations adverses.

En effet, le développement et l'introduction d'un nouveau produit font partie de la diversification, qui est une stratégie répandue dans le secteur manufacturier (Haug et Pidun, 2013). Pour Su et Tsang (2015), elle vise un changement d'orientation au sein de l'entreprise, soit en développant de nouveaux produits, soit en s'étendant sur un nouveau marché. Elle est adoptée pour compenser les pertes enregistrées avec un produit ou sur un marché affecté par la crise par le biais du profit provenant d'un autre produit ou d'un nouveau marché.

Ainsi, les récits examinés dans ce travail nous montrent que les femmes engagées dans des activités génératrices de revenus recourent à des stratégies d'adaptation ou de survie qui, en plus, peuvent être classées comme des innovations sociales. Les extraits successifs suivants en donnent une illustration :

Mon activité me permet de vivre. C'est vrai que la grande activité n'y est plus, mais, en ce qui concerne la production des masques, Dieu est en train de faire. (madame Aubierge)

Avant, les vendeuses du marché achetaient toute la planche et, en deux jours, sinon trois, elles avaient récupéré toute la production. Maintenant, elles achètent juste la moitié de la planche et je me débrouille à vendre le reste, chaque jour, sur le chemin de retour à la maison. Lorsque je ne vends pas tout, je le fais le matin en venant au jardin. (madame Raguie)

Avant la crise, nous avions un réseau de clients et la livraison se faisait à domicile et dans les marchés, notamment ceux de Texaco et Massengo.
Avec la limitation des mouvements due à la pandémie, nous avons développé de nouveaux circuits de livraison aux alentours des sites de production à la conquête des zones d'accès difficiles telles que Trois-Poteaux, à Massengo. (madame Julie)

Enfin, les deux stratégies fonctionnent bien puisqu'elles sont répandues chez les couturières pour la fabrication de masques artisanaux et chez les maraichères pour la vente ambulante de produits, même après le confinement.

\section{Flexibilité dans la gestion de la main-d'cuure}

En ce qui concerne les stratégies liées à la maind'œuvre, celles-ci permettent aux femmes de s'adapter à un environnement complexe et dynamique (Way, Wright et Tracey, 2013; Camps, Oltra, AldàsManzano, Buenaventura-Vera et Torres-Carbballo, 2015). Cette flexibilité est indispensable dans un contexte de crise comme celui du coronavirus. À cet égard, les récits exploités dans ce travail suggèrent que les femmes entrepreneures recourent à la maind'œuvre en fonction du volume de leurs activités et du contexte de la crise.

Les femmes qui ont une épargne peuvent drainer, par le biais du salariat ou de paiement forfaitaire, la maind'œuvre disponible. C'est le cas de madame Julie, qui s'engage à recruter du personnel pour faire face à la crise. De même, madame Justine paie le service d'un transporteur à la brouette pour acheminer ses achats jusqu’à la maison. À l'opposé, celles qui ne peuvent pas recourir à ces deux premières formes développent une troisième forme de recours, qui est l'implication des membres de la famille. C'est le cas de madame Jacqueline, qui a recours à ses enfants pour vendre ses beignets et de madame Inès, qui fait appel à son frère pour l'aider dans la vente de pain.

Ainsi, la flexibilité dans la gestion des ressources humaines apparait comme une capacité grâce à laquelle les femmes peuvent facilement s'adapter aux changements des contingences environnementales (Camps et collab., 2015; Sekhar, Patwardhan et Vyas, 2016). Bien que cette stratégie ne soit pas une innovation sociale, elle aide bien les femmes à poursuivre leurs activités en période de crise, où les actifs incorporels tels que les connaissances et d'autres actifs corporels jouent un rôle important (Aryee, Walumbwa, Seidu et Otaye, 2016). 


\section{Conclusion et implications de politiques}

L'objectif de ce travail était d'identifier les stratégies développées par les femmes à la tête de microentreprises et de vérifier si celles-ci sont des innovations sociales. Pour ce faire, des récits de vie ont été recueillis pendant la période du confinement pandémique couvrant les mois de mars et d'avril 2020. L'analyse de ces récits a montré que les stratégies développées par les femmes sont ambidextres. Elles associent aussi bien les stratégies d'exploitation que d'exploration. Les stratégies d'exploitation ont porté sur les déplacements, sur la flexibilité dans la gestion de la main-d'œuvre et sur l'ajustement des jours et des horaires de travail pour maintenir un certain niveau d'activité en pleine crise pandémique. Les stratégies d'exploration, quant à elles, ont porté sur la recherche d'une nouvelle clientèle et sur le développement de nouveaux produits en période de crise.

En prenant appui sur les critères de Bund et ses collègues, (2013), l'analyse a montré que toutes les stratégies développées par les femmes ne sont pas des innovations sociales. Dans le cas des récits de vie exploités dans cet article, seules la production des masques artisanaux et la vente ambulante de produits maraichers peuvent être considérées comme des innovations sociales. Les stratégies relatives à la gestion des ressources humaines sont certes indispensables à la survie des activités, mais ne peuvent pas être classées comme des innovations sociales au sens de Bund et ses collaborateurs, (2013).

En somme, l'ambidextérité est une réalité dans les microentreprises des femmes, notamment en période de crise pandémique. Ce résultat suggère que la capacité à développer de façon simultanée des activités d'exploitation et d'exploration a été déterminante dans la poursuite des activités des femmes pendant la crise. Néanmoins, les femmes à la tête de microentreprises doivent également cultiver l'innovation pour faire face aux changements qui se produisent constamment dans leur environnement de travail. C'est pourquoi elles doivent garder à l'esprit le rôle important que joue le développement de la flexibilité dans la pratique de leur activité.

En effet, ces entrepreneures doivent commencer à employer plus rigoureusement des formules de flexibilité fonctionnelle qui contribuent au succès de l'entreprise. Il s'agit d'une conception de la flexibilité placée dans le paradigme des capacités dynamiques de l'organisation, ce qui faciliterait le développement d'un apprentissage ambidextre. Par conséquent, elle faciliterait aussi la capacité de l'organisation à être efficace en exploitant les connaissances qu'elle possède ainsi que la capacité d'apprendre à s'adapter aux nouvelles exigences imposées par son environnement.

Ainsi, il est nécessaire d'insister, en matière de structures d'encadrement des femmes entrepreneures, sur la flexibilité comportementale, c'est-àdire l'aptitude des femmes à développer la capacité à adapter leurs actions aux nouvelles situations qui pourraient survenir dans leur environnement. Ces femmes ne pourront atteindre cette flexibilité comportementale qu'en ayant développé la flexibilité des compétences. En d'autres termes, les femmes à la tête d'une microentreprise doivent être polyvalentes et développer de nouvelles compétences afin d'accomplir les différentes tâches et/ou fonctions qu'exige l'ambidextrie et qui les mèneront à l'innovation sociale.

\section{REMERCIEMENTS}

Nous adressons nos sincères remerciements à Steffie Raynica Mboulou, Jacqueline Offélé, Roland Didier Frédéric Bany et Igor Bambi, tous chercheurs au Laboratoire de recherches et d'études économiques et sociales (LARES), pour leur précieuse aide lors de la phase de recueil des récits de vie en période de confinement.

\section{NOTES}

1 Les cinq forces évoquées sont : 1) le pouvoir de négociation des clients, 2) le pouvoir de négociation des fournisseurs, 3) la menace des produits de substitution, 4) la menace d'entrants potentiels sur le marché et 5) l'intensité de la rivalité entre les concurrents.

2 Les activités des femmes en microentreprise sont dominées par le commerce et par l'agriculture.

3 Au Congo, cette expression signifie que Dieu ne cesse de nous apporter son secours. 


\section{RÉFÉRENCES}

Ahammad, F. M., Mook Lee, S., Malul, M. et Shoham, A. (2015). Behavioral ambidexterity: The impact of incentive schemes on productivity, motivation, and performance of employees in commercial banks, Human Resource Management, 54(1), 45-62. https://doi.org/10.1002/hrm.21668

Aryee, S., Walumbwa, F. O., Seidu, E. Y. et Otaye, L. E. (2016). Developing and leveraging human capital resource to promote service quality testing a theory of performance. Journal of Management, 42(2), 480-499. https://doi.org/10.1177/0149206312471394

Asare, R., Akuffobea, M., Quaye, W. et Atta-Antwi, K. (2015). Characteristics of micro, small and medium enterprises in Ghana: Gender and implications for economic growth. African Journal of Science, Technology, Innovation and Development, 7(1), 26-35. https://doi.org/10.1080/20421338.2014.979651

Atuahene-Gima, K. (2005). Resolving the capability-rigidity paradox in new product innovation. Journal of Marketing, 69(4), 61-83. https://doi.org/10.1509/jmkg.2005.69.4.61

Barney, J. B. (1991). Firm resources and sustained competitive advantage. Journal of Management, 17(1), 99-120. https://doi.org/10.1177/014920639101700108

Berry, A., Rodriguez, E. et Sandee, H. (2001). Small and medium enterprise dynamics in Indonesia. Bulletin of Indonesian Economic Studies, 37(3), 363-368. https://doi.org/10.1080/00074910152669181

Bund, E., Hubrich, D.-K., Schmitz, B., Mildenberger, G. et Krlev. G. (2013a). Blueprint of social innovation metrics contributions to an under- standing of opportunities and challenges of social innovation measurement. A deliverable of the project TEPSIE. Brussels: European Commission. Repéré à https://archiv.ub.uni-heidelberg.de/ volltextserver/18700/1/D2.4_final.pdf

Bruner, J. (1986). Actual Minds, Possible Worlds. Cambridge, Mass: Harvard University Press.

Bryan, L. et Farrell, D. (2008). Leading through uncertainty. McKinsey Quarterly. Repéré à http://www.mckinseyquarterly.com/Leading_through_uncertainty_2263

Camps J., Oltra, V., Aldás-Manzano, J., Buenaventura-Vera, G. et Torres-Carballo, F. (2015). Individual performance in turbulent environments: The role of organizational learning capability and employee flexibility. Human Resource Management, 55(3), 363-383. https://doi.org/10.1002/hrm.21741

Cantarello Nosella, A. S. et Filippini, R. (2012). The intellectual structure of organizational ambidexterity: A bibliographic investigation into the state of the art. Strategic Organization, 10(4), 450-465. https://doi.org/10.1177/1476127012457979

Cao, Q., Gedajlovic, E. et Zhang, H. (2009). Unpacking organizational ambidexterity: Dimensions, contingencies, and synergistic effects. Organization Science, 20(4), 781-796. https://doi.org/10.1287/orsc.1090.0426

Chebbi, H., Yahiaoui, D., Vrontis, D. et Thrassou, A. (2015). Building multiunit ambidextrous organizations: A transformative framework. Human Resource Management, 54(1), 115-177. https://doi.org/10.1002/hrm.21662

Christensen, C. M. (1997). The innovator's dilemma: When new technologies cause great firms to fail. Boston, MA: Harvard Business School Press.

Cooper, P. et McIntyre, D. (1993). Commonality in teachers' and pupils' perceptions of effective classroom learning. British Journal of Educational Psychology, 63(3), 381-399. doi:10.1111/j.2044-8279.1993.tb01066.x

Dedee, J. et Vorhies, D. (1998). Retrenchment activities of small firms during economic downturn: An empirical investigation. Journal of Small Business Management, 36(3), 46-61.

Duncan, R. B. (1976). The ambidextrous organization: Designing dual structures for innovation. Dans R. H. Kilmann, L. R. Pondy et D. Slevin (dir.), The management of organization design: Strategies and implementation (p. 167-188). New York, NY: North Holland.

Eisenhardt, K. M. et Martin, J. A. (2000). Dynamic capabilities: What are they? Strategic Management Journal, 21(10-11), 1105-1121. https://doi.org/10.1002/1097-0266

Farjoun, M. (2010). Beyond dualism: Stability and change as a duality. Academy of Management Review, 35(2), 202-225. https://doi.org/10.5465/amr.35.2.zok202 
Gulati, R. etPuranam, P. (2009). Renewal Through Reorganization: The V alue of Inconsistencies Between Formal and Informal Organization. Organization Science, 20(2), 422-440. doi:10.1287/orsc.1090.0421

Gupta, A. K., Smith, K. G. et Shalley, C. E. (2006). The interplay between exploration and exploitation. Academy of Management Journal, 49(4), 693-706. https://doi.org/10.5465/amj.2006.22083026

Hahn, T., Pinkse, J., Preuss, L. et Figge, F. (2016). Ambidexterity for corporate social performance. Organization Studies, 37(2), 213-235. https://dx.doi.org/10.1177/0170840615604506

Hall, C. et Harvie, C. (2003). A comparison of the performance of SMEs in Korea and Taiwan: Policy implications for turbulent times (Mémoire de maitrise non publié), University of Wollongong, Wollongong, Australie.

Haubert, M. (1999). L’application des politiques libérales dans le secteur agraire et le rôle des paysans comme entrepreneurs. Revue Tiers Monde, XL(157), 87-106. https://doi.org/10.3406/tiers.1999.5368

Haug, J et Pidun, U. (2013). Diversification and Competitive Advantage: A Risk-Based Explanation. Academy of Management Annual Meeting Proceedings, 2013(1), 13418-13418. doi: 10.5465/AMBPP.2013.13418abstract

He, Z. L. et Wong, P. K. (2004). Exploration vs. exploitation: An empirical test of the ambidexterity hypothesis. Organization Science, 15(4), 481-494. https://doi.org/10.1287/orsc.1040.0078

Hodorogel, R. G. (2009). The economic crisis and its effects on SMEs. Theoretical and Applied Economics, 5(534), 79-88. Repéré à http://www.store.ectap.ro/articole/389.pdf

Hugon, P. (1996). Incertitude, précarité et financement local : le cas des économies africaines. Revue Tiers Monde, 145, 13-40. https://doi.org/10.3406/tiers.1996.5026

Koubaa, S. (2017). L'ambidextrie pour comprendre l'action de l'entrepreneur. Projectics/Proyéctica/Projectique, 1(16), 31-50. Repéré à https://www.cairn.info/revue-projectique-2017-1-page-31.htm

Lesteven, A. (1985). L'économie sociale familiale dans le développement rural : quelques réflexions sur la situation de l'Afrique noire francophone. UNESCO : Science and Technology Education Document Series, 14.

Repéré à https://unesdoc.unesco.org/ark:/48223/pf0000064167

Lynn, M. (2009, 17 mai). The new capitalism. Sunday Times, p. 4.

March, J. G. (1991). Exploration and exploitation in organizational learning. Organization Science, 2(1), 71-87. Repéré à https://www.jstor.org/stable/2634940

Michael, S. C. et Robbins, K. D. (1998). Retrenchment among small manufacturing firms during recession. Journal of Small Business Management, 36(3), 35-45.

Missié J. P. (2005). Dépérissement de l'Etat et stratégies de survie en Afrique centrale : le cas des exclus sociaux au Congo Brazzaville. Dans Mukawa D. P. et Tchouassi G. (dir.), Afrique Centrale : crises économiques et mécanismes de survie (p. 315-325), CODESRIA, Dakar.

Mom, T. J. M., van den Bosch, F. A. J. et Volberda, H. W. (2009). Understanding variation in managers' ambidexterity: Investigating direct and interaction effects of formal structural and personal coordination mechanisms. Organization Science, 20(4), 812-828. http://dx.doi.org/10.1287/orsc.1090.0427

Monnet, J. (2006). Le commerce de rue, ambulant ou informel et ses rapports avec la métropolisation : une ébauche de modélisation. Autrepart, 3(39), 93-109. https://doi.org/10.3917/autr.039.0093

MPMEASI et INS. (2017). Recensement des très petites, petites, moyennes entreprises et des artisans : rapport principal. Ministre des petites et moyennes entreprises, de l'artisanat et du secteur informel et Institut National de la Statistique. Brazzaville: MPMEASI et INS.

Narjoko, D. et Hill, H. (2007). Winners and losers during a deep economic crisis: Firm-level evidence from Indonesian manufacturing. Asian Economic Journal, 21(4), 343-368. https://doi.org/10.1111/j.1467-8381.2007.00261.x

Nugent, J. et Yhee, S. (2002). Small and medium enterprises in Korea: Achievements, constraints and policy issues. Small Business Economics, 18, 85-119. https ://doi.org/10.1023/A :1015181911497 
Organisation de coopération et de développement économiques (OCDE). (2004, 3-5 juin). Promouvoir l'entrepreneuriat et les PME innovantes dans une économie mondiale : vers une mondialisation plus responsable et mieux partagée. Communication présentée lors de la $2^{e}$ Conférence de l'OCDE des ministres en charge des petites et moyennes entreprises (PME), Istanbul, Turquie. Repéré à http ://www.oecd.org/fr/industrie/pme/31946170.pdf

O'Reilly, C. A. et Tushman, M. L. (2008). Ambidexterity as a dynamic capability: Resolving the innovator's dilemma. Research in Organizational Behavior, 28, 185-206. https://doi.org/10.1016/j.riob.2008.06.002

Penrose, R. (1989). The emperor's new mind: Concerning computers, minds, and the laws of physics. Oxford, R.-U. : Oxford University Press.

Porter, M. E. (1980a). Competitive strategy: Techniques for analyzing industries and competitors. New York, NY : Free Press.

Prieto, I. M. et Pérez Santana, M. (2012). Building ambidexterity: The role of human resource practices in the performance of firms from Spain. Human Resource Management, 51(2), 189-211. https://doi.org/10.1002/hrm.21463

Raisch, S. et Birkinshaw, J. (2008). Organizational ambidexterity: Antecedents, outcomes, and moderators. Journal of

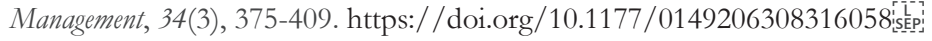

Rumelt, R. P. (1984). Towards a strategic theory of the firm. Dans R. B. Lam (dir.), Competitive strategic management (p. 566-570). Englewood Cliffs, NJ: Prentice Hall.

Sato, Y, (2000). How did the crisis affect small and medium-sized enterprises? From a field study of the metal-working industry in Java. The Developing Economies, 38(4), 572-595. https://doi.org/10.1111/j.1746-1049.2000.tb00891.x

Sekhar, C., Patwardhan, M. et Vyas, V. (2016). A study of HR flexibility and firm performance: A perspective from IT industry. Global Journal of Flexible Systems Management, 17(1), 57-75. https://doi.org/10.1007/s40171-015-0120-2

Smith, W. K. et Lewis, M. W. (2011). Toward a theory of paradox: A dynamic equilibrium model of organizing. Academy of Management Review, 36(2): 381-403. doi: 10.5465/AMR.2011.59330958

Smith, W. K. et Tushman, M. L. (2005). Managing strategic contradictions: A top management model for managing innovation streams. Organization Science, 16, 522-536. https://doi.org/10.1287/orsc.1050.0134

Su, W. et Tsang, E. W. K. (2015). Product diversification and financial performance: The moderating role of secondary stakeholders. Academy of Management Journal, 58(4), 1128-1148. https://doi.org/10.5465/amj.2013.0454

Teece, D. J. (2007). Explicating dynamic capabilities: The nature and microfoundations of (sustainable) enterprise performance. Strategic Management Journal, 28(13), 1319-1350. https://doi.org/10.1002/smj.640

ter Wengel, J. et Rodriguez, E. (2006). SME export performance in Indonesia after the crisis. Small Business Economics, 26, 25-37. https://doi.org/10.1007/s11187-004-6491-y

Tripsas, M. (1997). Unraveling the process of creative destruction: Complementary assets and incumbent survival in the typesetter industry. Strategic Management Journal, 18(Summer Special Issue), 119-142.

Repéré à https://www.jstor.org/stable/3088213

Turner, N., Swart, J. et Maylor, H. (2013). Mechanisms for managing ambidexterity: A review and research agenda. International Journal of Management Reviews, 15(3), 317-332. https://doi.org/10.1111/j.1468-2370.2012.00343.x

Úbeda-García, M., Claver-Cortés, E., Marco-Lajara, B. et Zaragoza-Sáez, P. (2017). Human resource flexibility and performance in the hotel industry: The role of organizational ambidexterity. Personnel Review, 46(4), 824-846. https://doi.org/10.1108/PR-12-2015-0315

Way, S. A., Wright, P. M. et Tracey, J. B. (2013). HR flexibility and firm performance: The cross-level moderating effect of industry dynamism. Academy of Management Proceedings, 1. https://doi.org/10.5465/AMBPP.2013.10607abstract

Wernerfelt, B. (1984). A resource-based view of the firm. Strategic Management Journal, 5(2), 171-180. https://doi.org/10.1002/smj.4250050207

Wooldridge, B. et Floyd, S. W. (1990). The strategy process, middle management involvement, and the organizational performance. Strategic Management Journal, 11(3), 231-241. https://doi.org/10.1002/smj.4250110305

Zimmermann, A., Raisch, S. et Birkinshaw, J. (2015). How is ambidexterity initiated? The emergent charter definition process. Organization Science, 26(4), 1119-1139. https://doi.org/10.1287/orsc.2015.0971 


\section{Annexe}

\section{Récit de Raguie : une agricultrice de la ceinture maraichère de Brazzaville}

"Je travaille à 100 fils. Je suis maraichère. Nous avons commencé le maraichage depuis longtemps. Depuis la crise du coronavirus (COVID-19), nous ne travaillons plus assez. Nous travaillons très peu parce que nous avons peu de temps. Nous venons à pied et nous arrivons au lieu de travail tard et le temps de travail est réduit. Nous quittons la maison à $7 \mathrm{~h}$ et nous arrivons au travail à $10 \mathrm{~h}$. À $15 \mathrm{~h}$, nous devons déjà quitter le marché. Comme vous pouvez le constater, le temps de travail est réduit. Vraiment, même vendre devient difficile : la production est faible et les produits à vendre ont baissé, tout comme les prix. Il en est ainsi parce que le marché a lieu seulement trois jours dans la semaine (lundi, mercredi et vendredi). Nos clients n'achètent plus assez, car s'ils ne vendent pas tout le jour du marché, ils vont enregistrer des pertes, car, le jour suivant, il n’y a pas de marché et les produits se dégradent.

Avant, les vendeuses au marché achetaient toute la planche et, en deux jours, sinon trois, elles avaient récupéré toute la production. Maintenant, elles achètent juste la moitié de la planche et je me débrouille à vendre le reste, chaque jour, sur le chemin de retour à la maison. Lorsque je ne vends pas tout, je le fais le matin en venant au jardin.

Avant la crise du coronavirus, nous vendions bien notre production; nous étions bien. Mais maintenant, il y a un décalage dans la vente et dans le travail. Parfois, nous avons mal aux pieds. Nous venons et rentrons à pied, donc c'est très difficile. Cette activité nous permet de prendre soin de la famille, des enfants. Le maraichage est une bonne activité. Mais, maintenant que nous ne travaillons pas assez, l'entretien des enfants devient difficile parce que nous vendons seulement comme ça. Nous pratiquons des prix bas pour que les clients achètent pour écouler les produits. Comme mon mari ne travaille plus, nous vivons uniquement du maraichage et l'entretien des enfants devient difficile. »

\section{Récit de madame Jacqueline : une femme engagée dans une activité de fabrication et de vente des beignets}

«Je m'appelle Jacqueline et mon activité consiste en la fabrication et la vente de beignets. J'exerce cette activité depuis trois ans. En temps normal, pour la fabrication des beignets, j'achète un demi-sac de farine, un demi-sac de sucre, un bidon d'huile, du bois de chauffe, la levure et du papier d'emballage. Je me procurais ces intrants à bon prix avant la crise. Maintenant, les prix de ces intrants ont augmenté. Je n'arrive plus à acheter un demi-sac de farine parce que mon budget ne me le permet plus. Je me contente d'un quart de sac de farine pour mon activité quotidienne. Il n'y a que le déplacement qui ne me coûte pas grand-chose, car je m'approvisionne au même endroit et c'est proche de la maison.

Avant, je produisais beaucoup. Avec un demi-sac de farine, je pouvais faire une recette de 30 000, voire 35000 francs CFA par jour. Les clients achetaient aussi, donc je pouvais vendre tous mes beignets le même jour. Maintenant, avec un quart de sac de farine, je n'arrive pas à écouler toute ma production. Il y a beaucoup d'invendus. Pour limiter les pertes, je suis obligée de vendre les beignets à mes voisins en me déplaçant de parcelle en parcelle. Le bénéfice ne répond plus. Depuis le début de la crise, le bénéfice n'est plus important. Je ne peux plus épargner comme avant. Cette crise nous pousse même à nous endetter pour continuer à faire notre activité.

Dans l'exercice de mon activité, je suis en contact avec beaucoup de clients. Je peux dire que j'ai la maladie devant moi tous les jours puisque je vends. Pour éviter la contamination au coronavirus, j’ai acheté des bavettes et j'ai de l'eau en permanence pour que mes clients se lavent les mains, d'autant plus que certains mangent sur place. 
Cette activité me permet de prendre en charge certaines dépenses de la maison. Maintenant, c'est difficile parce que mon mari ne travaille plus. Il n'a rien et je dois prendre en charge toutes les dépenses de la maison et c'est lourd pour moi. Tous les jours, j'exécute les mêmes tâches à la maison. C'est au niveau de mon activité que les jours et les heures ont changé. Déjà, je n'exerce mon activité que trois jours dans la semaine (lundi, mercredi et vendredi). Cela tue mon activité parce que trois jours, c'est peu et j'ai une grande charge. Il n'est pas possible d'épargner dans ces conditions. Je suis engagée dans une ristourne et maintenant c'est difficile d'honorer cet engagement.

Sur le plan de la famille, je bénéficie de l'aide des enfants dans l'exercice de mon activité. Les enfants m'aident beaucoup. Ils vendent les beignets pendant que je fais la production. Maintenant, ils ne peuvent plus m'aider à cause du coronavirus, qui nous impose de les protéger. Mon mari m'aidait aussi financièrement. Mais aujourd'hui, il ne travaille plus et ne peut pas me soutenir. En ce moment, je pleure, tout comme lui. Personne ne peut aider l'autre; c'est difficile. Nous sommes comme en guerre parce que les activités sont inexistantes. C'est comme si les gens avaient déménagé. La ville n’a plus personne; chacun est dans son coin. Sur les portails, il est écrit Restez, chę vous (rire). Le pays est en péril. »

\section{Récit de madame Inès : une vendeuse de pain}

«Je suis madame Inès. Je tiens une boutique de vente de pain à Moukondo. J'exerce cette activité depuis trois mois. Mon activité consiste à vendre du pain pour le compte d'une boulangerie, qui me livre le pain le matin et l'après-midi. Avant le confinement, la boutique était ouverte de $6 \mathrm{~h}$ à $22 \mathrm{~h}$. Maintenant, elle n'est ouverte que de 8 h à 19 h 30 à cause du couvre-feu, qui commence à 20 h. En dépit de ce changement, les ventes ont augmenté depuis la crise sanitaire. La recette est passée, en moyenne, de 100000 à 150000 francs CFA.

Mon activité se déroule bien, car je n'ai aucun problème de déplacement parce que j'habite dans le quartier. J'ai pris des précautions pour éviter la COVID-19. Je fais obligation aux clients de se laver les mains à l'entrée de la boutique et je fais respecter la distanciation sociale. J'ai des gants pour prendre l'argent et une pince pour éviter de toucher les pains avec mes mains. L'activité est très rentable depuis qu'il y a le confinement. Le respect des heures du couvre-feu fait que la boulangerie me livre le pain à $8 \mathrm{~h}$, alors que les clients y sont déjà, donc cela crée un peu de désordre.

Dans l'exercice de mon activité, en pleine crise de la COVID-19, je bénéficie du soutien de ma famille, notamment mon frère. À cause de la crise, les deux filles qui travaillent chez moi sont confinées. Je tiens la boutique moi-même et, quand je me déplace pour une heure ou deux, c'est mon frère qui vient me suppléer.

J'ai ouvert la boutique le 19 février 2020. En temps normal, les gens vaquent à leurs occupations et nombreux sont ceux qui ne connaissaient pas la boutique. Je peux dire que j'ai la maladie devant moi tous les jours puisque je vends. La crise de la COVID-19 a été donc une opportunité pour lancer mon activité. » 